ARTICLE

https://doi.org/10.1038/s41467-020-16532-x

\title{
OPEN
}

\section{Spherical trihedral metallo-borospherenes}

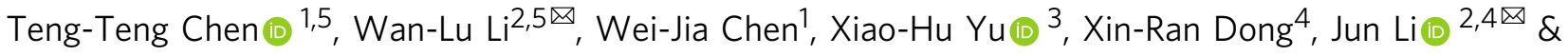 \\ Lai-Sheng Wang (1) ${ }^{1 凶}$
}

The discovery of borospherenes unveiled the capacity of boron to form fullerene-like cage structures. While fullerenes are known to entrap metal atoms to form endohedral metallofullerenes, few metal atoms have been observed to be part of the fullerene cages. Here we report the observation of a class of remarkable metallo-borospherenes, where metal atoms are integral parts of the cage surface. We have produced $\mathrm{La}_{3} \mathrm{~B}_{18}{ }^{-}$and $\mathrm{Tb}_{3} \mathrm{~B}_{18}{ }^{-}$and probed their structures and bonding using photoelectron spectroscopy and theoretical calculations. Global minimum searches revealed that the most stable structures of $\mathrm{Ln}_{3} \mathrm{~B}_{18^{-}}$are hollow cages with $D_{3 h}$ symmetry. The $B_{18}$-framework in the $\operatorname{Ln}_{3} B_{18}{ }^{-}$cages can be viewed as consisting of two triangular $B_{6}$ motifs connected by three $B_{2}$ units, forming three shared $B_{10}$ rings which are coordinated to the three $\mathrm{Ln}$ atoms on the cage surface. These metalloborospherenes represent a new class of unusual geometry that has not been observed in chemistry heretofore.

\footnotetext{
${ }^{1}$ Department of Chemistry, Brown University, Providence, RI 02912, USA. ${ }^{2}$ Department of Chemistry and Key Laboratory of Organic Optoelectronics \& Molecular Engineering of Ministry of Education, Tsinghua University, 100084 Beijing, China. ${ }^{3}$ Institute of Theoretical and Computational Chemistry, Shaanxi Key Laboratory of Catalysis, School of Chemical \& Environment Sciences, Shaanxi University of Technology, 723000 Hanzhong, China. ${ }^{4}$ Department of Chemistry, Southern University of Science and Technology, 518055 Shenzhen, China. ${ }^{5}$ These authors contributed equally: Teng-Teng Chen, Wan-Lu Li.

凶email: wanluli0716@gmail.com; junli@tsinghua.edu.cn; lai-sheng_wang@brown.edu
} 
T he electron deficiency of boron often leads to electron delocalization and the violation of the octet rule in boron compounds and three-dimensional (3D) cage units in different bulk boron allotropes ${ }^{1-3}$. Because of the strong boron-boron bonding, there were speculations about the formation of boron nanotubes composed of a triangular boron lattice ${ }^{4,5}$, after the discovery of carbon nanotubes. The triangular boron lattice can be viewed as a graphene-like sheet with the filling of a boron atom in each $\mathrm{B}_{6}$ hexagon. Further theoretical calculations revealed, however, that triangular lattices with hexagonal vacancies were more stable and more suitable to construct boron nanotubes ${ }^{6,7}$. In the meantime, combined spectroscopic and theoretical studies have shown that size-selected boron clusters all have 2D structures with delocalized multi-center bonding within the cluster plane ${ }^{8-11}$. The discovery of the hexagonal $2 \mathrm{D} \mathrm{B}_{36}$ clusters provided the first experimental evidence of the viability of atom-thin boron nanostructures with hexagonal vacancies, named as borophene akin to graphene ${ }^{12}$. Borophenes have been recently synthesized using atomic vapor deposition on $\mathrm{Ag}(111)$ substrates ${ }^{13,14}$, forming a new class of synthetic $2 \mathrm{D}$ nanomaterials ${ }^{15}$. The analogy between nanostructures made of boron and carbon has been further extended when the $\mathrm{B}_{40}$ and $\mathrm{B}_{39^{-}}$clusters were found to have global minimum cage structures $^{16,17}$, i.e. borospherenes analogous to the fullerenes. Fullerenes are known to form endohedral metallofullerenes for alkali, alkali earth, lanthanide, and actinide elements ${ }^{18,19}$, albeit not for transitions metals. Heterofullerenes in which one carbon atom is substituted by a transition metal atom have been observed in the gas phase, but the metal substitution induces large local structural distortions and such heterofullerenes have not been synthesized in the bulk ${ }^{20-22}$. The first cage cluster made of multiple metal atoms and carbons was proposed to be $\mathrm{Ti}_{8} \mathrm{C}_{12}+$ (metallocarbohedrene) ${ }^{23}$. However, subsequent theoretical calculations showed that the metallocarbohedrene is not stable and the global minimum of $\mathrm{Ti}_{8} \mathrm{C}_{12}{ }^{+}$consisted of a tetrahedral, closepacked $\mathrm{Ti}_{8}$ clusters coordinated by six $\mathrm{C}_{2}$ units on the cluster surface $^{24}$. In the present article, we report the first observation of a class of metallo-borospherenes, hollow cage clusters consisting of three lanthanide $(\mathrm{Ln})$ atoms and 18 boron atoms $\left(\operatorname{Ln}_{3} \mathrm{~B}_{18^{-}}\right)$.

Transition-metal-doped boron clusters were first found to form

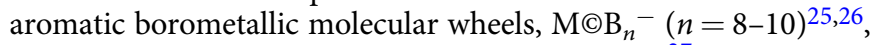
as well as metal-centered nanotubular structures ${ }^{27}$. More interestingly, it has been shown that transition metals can be an integral part of larger $2 \mathrm{D}$ boron clusters ${ }^{28}$, leading to the possibility of metallo-borophenes ${ }^{29}$. Lanthanide-doped boron clusters, however, have been found recently to form very different structures, due to both charge transfer interactions and strong $(d-p) \pi$ bonding ${ }^{30}$. For example, lanthanide-doped boron clusters do not form similar borometallic molecular wheels as the transition metals. Instead, they form inverse-sandwich-type structures for $\mathrm{Ln}_{2} \mathrm{~B}_{n}^{-}$clusters $(n=7-9)^{31,32}$. The most recent study indicates that the inverse-sandwich structure may extend to form lanthanide-boron nanowires ${ }^{33}$.

Here we report a joint photoelectron spectroscopy (PES) and quantum chemistry study of two tri-lanthanide-doped $\mathrm{B}_{18}$ clusters $\left(\mathrm{La}_{3} \mathrm{~B}_{18}{ }^{-}\right.$and $\left.\mathrm{Tb}_{3} \mathrm{~B}_{18}{ }^{-}\right)$, which are found to possess unprecedented $D_{3 h}$ cage structures with the Ln atoms being integral parts of the cage surface. These $D_{3 h}$ metallo-borospherenes belong to an unusual class of geometry known as spherical trihedron. The $B_{18}$ framework consists of two $B_{6}$ triangles connected by three $B_{2}$ units, forming three shared $B_{10}$ rings. The high stability of the spherical trihedral structures is derived from the strong interactions between the Ln atoms and the $B_{10}$ rings via charge transfer interactions and $d-p$ covalent bonding. Theoretical calculations show that the entire series of lanthanide elements $(\mathrm{Ln}=\mathrm{La}-\mathrm{Lu})$ can form spherical trihedral $\mathrm{Ln}_{3} \mathrm{~B}_{18}{ }^{-}$
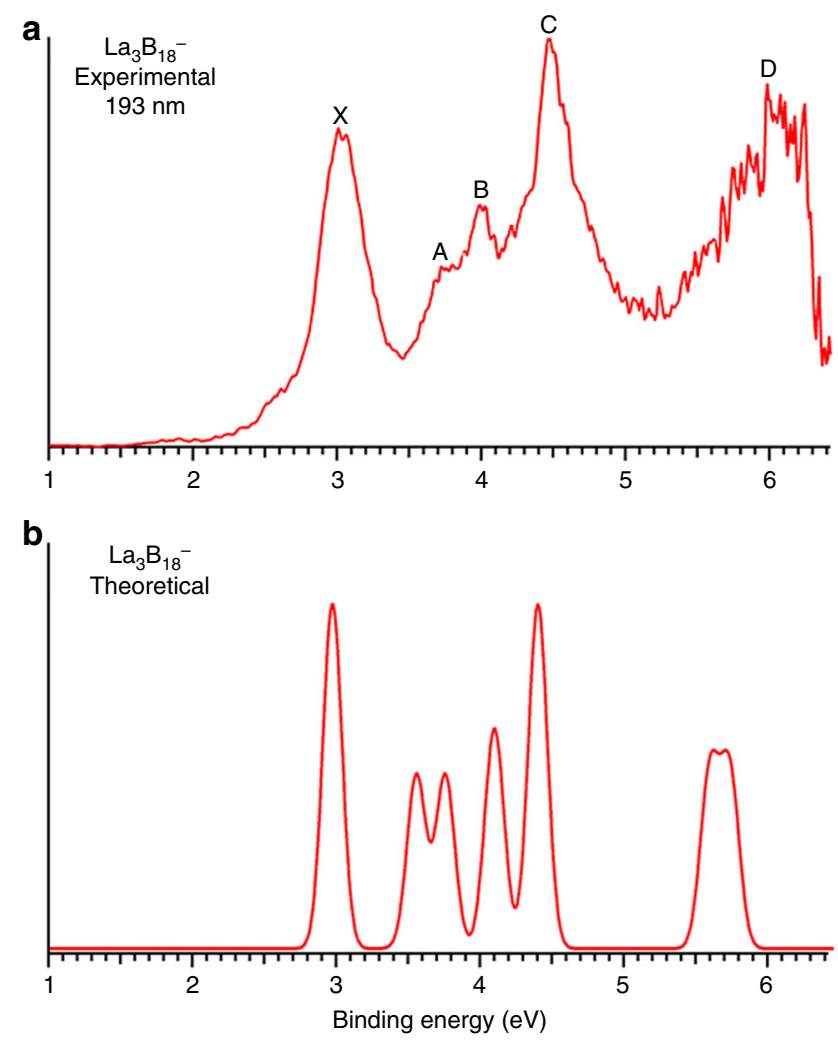

Fig. 1 Photoelectron spectrum of $\mathbf{L a}_{\mathbf{3}} \mathbf{B}_{\mathbf{1 8}}{ }^{-}$. a At $193 \mathrm{~nm}$. b The simulated spectrum.

metallo-borospherenes with tunable magnetic properties, making them a fascinating series of building blocks for new types of magnetic materials.

\section{Results and discussion}

Photoelectron spectroscopy. The PE spectrum at $193 \mathrm{~nm}$ was first measured for the $\mathrm{La}_{3} \mathrm{~B}_{18}{ }^{-}$cluster (Fig. 1a), which was found to exhibit a relatively simple pattern compared with that of the recently reported $\mathrm{La}_{3} \mathrm{~B}_{14}{ }^{-}$cluster ${ }^{33}$. This observation suggested that $\mathrm{La}_{3} \mathrm{~B}_{18}{ }^{-}$should possess a highly symmetric structure. Subsequently, we also obtained the spectrum of a late-Ln cluster $\mathrm{Tb}_{3} \mathrm{~B}_{18}{ }^{-}$(Fig. 2a) and observed a spectral pattern, exhibiting some similarities to that of $\mathrm{La}_{3} \mathrm{~B}_{18}{ }^{-}$and suggesting that these two Lndoped boron clusters should have similar structures and chemical bonding. The well-resolved PES features of the $\mathrm{Ln}_{3} \mathrm{~B}_{18}{ }^{-}$clusters serve as electronic fingerprints to allow analyses of their structures and chemical bonding by comparing with theoretical calculations, as shown in Figs. 1b, 2b, and Supplementary Tables S1 and $\mathrm{S} 2$ for $\mathrm{Ln}=\mathrm{La}$ and $\mathrm{Tb}$, respectively.

The spectrum of $\mathrm{La}_{3} \mathrm{~B}_{18}{ }^{-}$displayed five well-resolved bands labeled as X, A, B, C, and D (Fig. 1a). The X band yielded the first vertical detachment energy (VDE) of $2.97 \mathrm{eV}$ for $\mathrm{La}_{3} \mathrm{~B}_{18}{ }^{-}$. The adiabatic detachment energy (ADE) for band $\mathrm{X}$ was evaluated from its onset to be $2.80 \mathrm{eV}$, which also represents the electron affinity (EA) of neutral $\mathrm{La}_{3} \mathrm{~B}_{18}$. The higher binding energy bands (A, B, C, and D) correspond to detachment transitions to the excited states of neutral $\mathrm{La}_{3} \mathrm{~B}_{18}$. The $\mathrm{A}$ band at $3.64 \mathrm{eV}$ was broad and not well resolved at $193 \mathrm{~nm}$, but it was slightly better resolved in the $266 \mathrm{~nm}$ spectrum (Supplementary Fig. 1). This broad spectral feature could be due to geometry changes upon electron detachment or overlapping detachment transitions. Band B at $4.01 \mathrm{eV}$ is sharper compared with band A (Fig. 1a). An intense and sharp band C at $4.43 \mathrm{eV}$ was clearly resolved in the $193 \mathrm{~nm}$ 

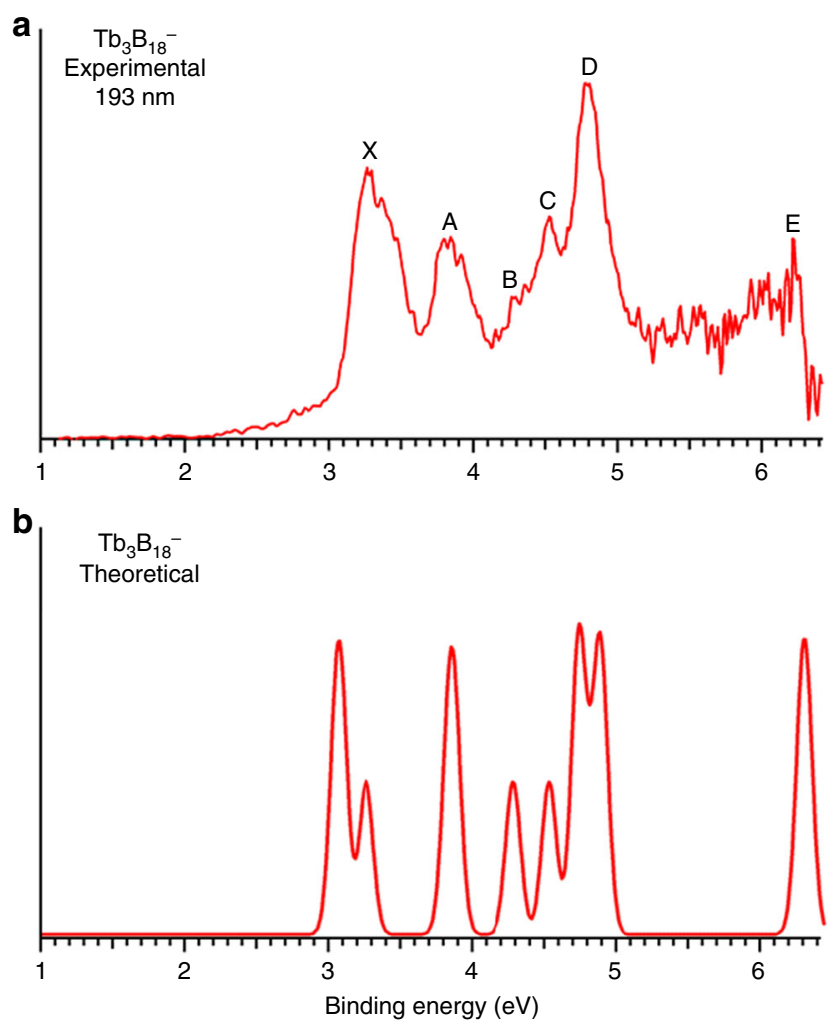

Fig. 2 Photoelectron spectrum of $\mathbf{T b}_{\mathbf{3}} \mathbf{B}_{\mathbf{1 8}}{ }^{-}$. a At $193 \mathrm{~nm}$. b The simulated spectrum.

spectrum. Following a large energy gap, a broad band (D) was observed above $\sim 5.5 \mathrm{eV}$. Due to the poor signal-to-noise ratio, band $\mathrm{D}$ was tentatively assigned for the sake of discussion.

The $\mathrm{PE}$ spectrum of $\mathrm{Tb}_{3} \mathrm{~B}_{18}{ }^{-}$showed five well-resolved peaks assigned as X, A, B, C, D (Fig. 2a). The $\mathrm{X}$ band gave rise to a VDE of $3.26 \mathrm{eV}$ for $\mathrm{Tb}_{3} \mathrm{~B}_{18}{ }^{-}$and an $\mathrm{ADE}$ of $3.13 \mathrm{eV}$, which is also the EA of neutral $\mathrm{Tb}_{3} \mathrm{~B}_{18}$. Band $\mathrm{A}$ was observed at a VDE of $3.84 \mathrm{eV}$, followed by three closely-lying bands (B, C, D). Band B at $4.28 \mathrm{eV}$ and band $\mathrm{C}$ at $4.52 \mathrm{eV}$ were relatively weak and closely spaced, whereas band $\mathrm{D}$ at $4.77 \mathrm{eV}$ was much more intense. Beyond $\sim 5 \mathrm{eV}$, the signal-to-noise ratio was poor and no obvious spectral bands were observed. Band E close to the threshold at a VDE of $\sim 6.2 \mathrm{eV}$ was tentatively labeled. The overall spectral pattern for $\mathrm{Tb}_{3} \mathrm{~B}_{18}{ }^{-}$exhibits some similarity to that of $\mathrm{La}_{3} \mathrm{~B}_{18}{ }^{-}$. In particular, the strong $\mathrm{X}$ and $\mathrm{D}$ bands in $\mathrm{Tb}_{3} \mathrm{~B}_{18}{ }^{-}$are similar to the strong $\mathrm{X}$ and $\mathrm{C}$ bands in $\mathrm{La}_{3} \mathrm{~B}_{18}{ }^{-}$. There is a large energy gap on the high binding energy side in both spectra. Similar spectral patterns could be an indication of similar structures, as have been observed for a series of dilanthanide clusters $\left(\operatorname{Ln}_{2} \mathrm{~B}_{8}{ }^{-}\right)^{31}$.

Global minimum structural searches. The low-lying isomers within $55 \mathrm{kcal} \mathrm{mol}^{-1}$ of the global minimum at the levels of PBE/ TZP and PBE0/TZP are presented in Supplementary Fig. 2. The global minimum of $\mathrm{La}_{3} \mathrm{~B}_{18}{ }^{-}$is a hollow cage with a closed-shell ground state $\left({ }^{1} A_{1}\right)$ and $D_{3 h}$ symmetry. This is a hetero-metalloborospherene, in which the three La atoms are integral parts of the cage surface, as shown in Fig. 3. All the other low-lying isomers are low-symmetry 3D structures, many of which are distorted cages. The highly symmetric global minimum $D_{3 h}$ metalloborospherene exhibits overwhelming stability relative to the other low-lying isomers: it is more stable than the nearest isomer with $C_{s}$ symmetry by $\sim 19 \mathrm{kcal} \mathrm{mol}^{-1}$ at the PBE/TZP and PBE0/TZP levels of theory. The $\mathrm{B}_{18}$ framework in the $\mathrm{La}_{3} \mathrm{~B}_{18}{ }^{-}$cage can be viewed as consisting of two $\mathrm{B}_{6}$ triangles linked together at their a

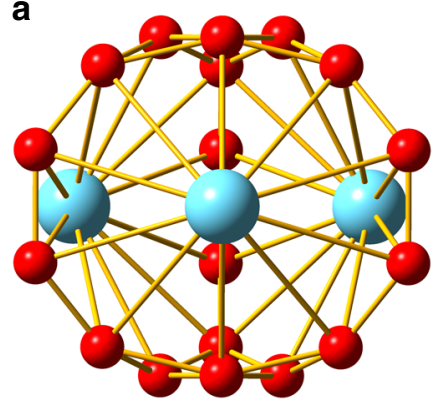

$$
\mathrm{La}_{3} \mathrm{~B}_{18}{ }^{-}\left(D_{3 h},{ }^{1} A_{1}\right)
$$

b

Fig. 3 The global minimum structure of $\operatorname{La}_{3} B_{18}{ }^{-}\left(D_{3 h},{ }^{1} A_{1}\right)$ at the PBEO/ TZP level. a The $C_{3}$ axis is along the page vertically. $\mathbf{b}$ The $C_{3}$ axis is perpendicular to the page.

three corners by three $B_{2}$ units, creating three shared $B_{10}$ rings along the $C_{3}$ axis. The three La atoms are coordinated by the three $\mathrm{B}_{10}$ rings, giving rise to the closed cage structure. The $\mathrm{La}_{3} \mathrm{~B}_{18}$ metallo-borospherene has an oblate shape with a diameter of $4.62 \AA$ along the $C_{3}$ axis (between the two $\mathrm{B}_{6}$ triangles) and $5.09 \AA$ encompassed by the three equatorial $\mathrm{La}$ atoms. The relevant bond lengths of the $\mathrm{La}_{3} \mathrm{~B}_{18}{ }^{-}$metallo-borospherene are shown in Supplementary Fig. 3a.

The hollow cage structure of $\mathrm{La}_{3} \mathrm{~B}_{18}{ }^{-}$was totally unexpected and the geometry is highly unusual. To further examine its stability and robustness, we performed ab initio molecular dynamics (AIMD) simulations at different temperatures, 300, 500, 700, and $1000 \mathrm{~K}$ (see Supplementary Fig. 4). We found that even at $1000 \mathrm{~K}$ the $\mathrm{La}_{3} \mathrm{~B}_{18}{ }^{-}$metallo-borospherene is dynamically stable for the $13 \mathrm{ps}$ duration of the simulations. At $1000 \mathrm{~K}$, the structure displayed a root-mean-square-deviation of $0.199 \AA$ and a maximum bond length deviation of $0.260 \AA$ during the simulations.

The similarity in their PE spectra suggested that the global minima of $\mathrm{Tb}_{3} \mathrm{~B}_{18}{ }^{-}$and $\mathrm{La}_{3} \mathrm{~B}_{18}{ }^{-}$should be similar. Because of the localized and nonbonding nature of the $4 f$ orbitals in $\mathrm{Tb}$, we optimized the $D_{3 h}$ structure for $\mathrm{Tb}_{3} \mathrm{~B}_{18}{ }^{-}$using the $4 f$-in-core pseudopotential ${ }^{34}$. The structural parameters of the $\mathrm{Tb}_{3} \mathrm{~B}_{18}{ }^{-}$ metallo-borospherene are similar to those for $\mathrm{La}_{3} \mathrm{~B}_{18}{ }^{-}$(Supplementary Fig. 3), except that the Tb-B and B-B bond lengths are all slightly shorter due to the smaller atomic radius of $\mathrm{Tb}$ as a result of the lanthanide contraction. Because of the use of the $4 f$ in-core pseudopotential, the spin state of the $\mathrm{Tb}_{3} \mathrm{~B}_{18}{ }^{-}$metalloborospherene was not determined from the geometry optimization. We performed broken symmetry calculations and compared the relative energies between the ferromagnetic and antiferromagnetic couplings of the $4 f$ electrons, as shown in Supplementary Table 3 for $\mathrm{Tb}_{3} \mathrm{~B}_{18}{ }^{-}$, as well as for $\mathrm{Pr}_{3} \mathrm{~B}_{18}{ }^{-}$. The relative energies due to the inter-atomic spin couplings of the unpaired $4 f$ electrons are relatively small, although the high spin ferromagnetic coupling seems to give the lowest energy in both cases. Hence, the spin multiplicity of the $\mathrm{Tb}_{3} \mathrm{~B}_{18}{ }^{-}$metallo-borospherene should be 19 (with 18 unpaired $4 f$ electrons).

Comparison between the experimental and theoretical results. To validate the $D_{3 h}$ cage structure for $\mathrm{La}_{3} \mathrm{~B}_{18}{ }^{-}$and $\mathrm{Tb}_{3} \mathrm{~B}_{18}{ }^{-}$, we calculated their ADEs and VDEs using the $\triangle$ SCF-TDDFT formalism. Figures $1 \mathrm{~b}, 2 \mathrm{~b}$ present the simulated spectra for the $D_{3 h}$ global minimum structures, in comparison with the experimental data. The computed $\mathrm{ADE} / \mathrm{VDE}_{1}$ at the $\mathrm{CCSD}(\mathrm{T})$ level are 2.828/2.972 eV for $\mathrm{La}_{3} \mathrm{~B}_{18}{ }^{-}$(Supplementary Table 4), in excellent agreement with the experimental data of $2.80 / 2.97 \mathrm{eV}$. As shown in Fig. 4, the valence MOs of $\mathrm{La}_{3} \mathrm{~B}_{18}{ }^{-}$are mainly of La-B $d-p$ and 


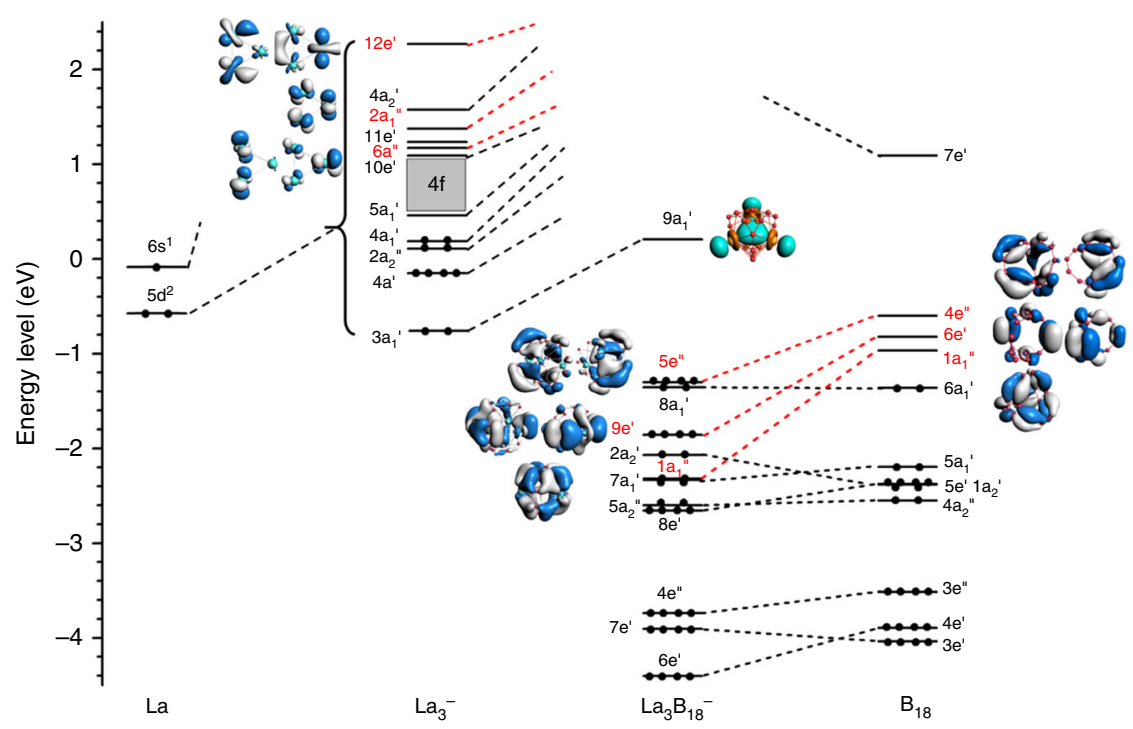

Fig. 4 The Kahn-Sham molecular orbital correlation diagram for $\mathbf{L a}_{\mathbf{3}} \mathbf{B}_{\mathbf{1 8}}{ }^{-}\left(\boldsymbol{D}_{\mathbf{3}},{ }^{\mathbf{1}} \boldsymbol{A}_{\mathbf{1}}\right)$. It shows the interactions between the $5 d$ orbitals of the three La atoms and the group orbitals of the $\mathrm{B}_{18}$ moiety.

B $s p$ characters. Because $\mathrm{La}_{3} \mathrm{~B}_{18}{ }^{-}$has a closed-shell configuration, single-electron removal from each molecular orbital (MO) yields one detachment channel, as shown in Supplementary Table 1. The computed VDEs for detachment from the $5 e^{\prime \prime} \mathrm{HOMO}$ $(2.972 \mathrm{eV})$ and $8 a_{1}{ }^{\prime}$ HOMO-1 $(2.987 \mathrm{eV})$ are very close to each other, in excellent agreement with the experimental VDE of the X band $(2.97 \mathrm{eV})$. In fact, each of the observed PES band corresponds to two detachment channels, as given in Supplementary Table 1, where the electron configurations and final state symmetries are also presented. The simulated spectral patterns and the experimental spectra are in excellent agreement, providing considerable credence for the $D_{3 h}$ cage global minimum for $\mathrm{La}_{3} \mathrm{~B}_{18}{ }^{-}$. We have also simulated the PE spectra for the next nine higher-lying isomers of $\mathrm{La}_{3} \mathrm{~B}_{18}^{-}$, as shown in Supplementary Fig. 5. None of these spectra fits the experimental spectrum, giving additional support for the $D_{3 h}$ global minimum structure.

The computed ADE/VDE for $\mathrm{Tb}_{3} \mathrm{~B}_{18}{ }^{-}$are 2.901/3.017 eV at the $\operatorname{CCSD}(\mathrm{T})$ level (Supplementary Table 4), slightly underestimated relative to the experimental data of $3.13 / 3.26 \mathrm{eV}$ probably due to the use of the $4 f$-in-core approximation as well as the incomplete account of electron correlations. Nevertheless, the theoretical results by not considering the $4 f$ electrons and detachment channels are still in very good agreement with the experimental data, as can be seen in Fig. 2 and Supplementary Table 2. These results are consistent with our previous observations that the detachment cross sections of the $4 f$ electrons are much weaker and the PE spectra of Ln-B binary clusters are dominated by the Ln-B $d-p$ and B sp detachment channels ${ }^{31,32}$.

Stabilities of the first metallo-borospherenes. The observation of the $\mathrm{La}_{3} \mathrm{~B}_{18}{ }^{-}$and $\mathrm{Tb}_{3} \mathrm{~B}_{18}{ }^{-}$cage clusters, in which the three $\mathrm{Ln}$ atoms are integral parts of the cage surface, is unprecedented. The two $\mathrm{B}_{6}$ triangles in the $D_{3 h}$ structure are reminiscent of the $\mathrm{B}_{40}$ borospherene $\mathrm{e}^{16}$, which consists of eight fused $\mathrm{B}_{6}$ triangles on a spherical surface. Hence, the $\operatorname{Ln}_{3} \mathrm{~B}_{18}{ }^{-}$cage clusters can be viewed as a new class of metallo-borospherenes. Networked metallofullerenes usually involve a single transition-metal atom substituting one carbon atom on the fullerene surface ${ }^{20-22}$. The incorporation of multiple metal atoms on the borospherene surface is due to the flexibility of the $2 \mathrm{D}$ boron network, which is a direct result of the electron deficiency of boron. It is interesting to note that the crystal structure of a Ni- $\mathrm{Zn}$ boride $\left(\mathrm{Ni}_{21} \mathrm{Zn}_{2} \mathrm{~B}_{24}\right)$ was shown to contain characteristic cages of $\mathrm{B}_{20}$ units, with an octahedral $\mathrm{Ni}_{6}$ cluster nested inside ${ }^{35}$. Our observation of the $\mathrm{Ln}_{3} \mathrm{~B}_{18}{ }^{-}$metallo-borospherenes represents the first isolated molecules of $\mathrm{Ln}-\mathrm{B}$ cages in the gas phase.

To understand the stability of these remarkable cage structures, we carried out fragment MO analyses by first considering the construction of the $\mathrm{B}_{18}$ framework in two different pathways and then its bonding with the three La atoms, as schematically shown in Fig. 5. Figure 5a shows one possible path to construct the $B_{18}$ framework by the fusion of three $B_{10}$ rings. Four of the boron atoms in each $\mathrm{B}_{10}$ ring are shared with the other $\mathrm{B}_{10}$ rings, which each coordinate to a $\mathrm{La}$ atom to form three shared $\mathrm{La} \odot \mathrm{B}_{10}$ units. This hypothetical formation pathway of $\mathrm{La}_{3} \mathrm{~B}_{18}{ }^{-}$can be expressed by the following steps:

$$
\begin{gathered}
3 \mathrm{~B}_{10} \rightarrow \mathrm{B}_{18}+3 \mathrm{~B}_{4} \quad \Delta E_{1}=120.1 \mathrm{kcal} \mathrm{mol}^{-1} \\
\mathrm{~B}_{18}+3 \mathrm{La} \rightarrow \mathrm{La}_{3} \mathrm{~B}_{18} \quad \Delta E_{2}=-655.8 \mathrm{kcal} \mathrm{mol}^{-1} \\
\mathrm{La}_{3} \mathrm{~B}_{18}+\mathrm{e}^{-} \rightarrow \mathrm{La}_{3} \mathrm{~B}_{18}^{-} \quad \Delta E_{3}=-68.8 \mathrm{kcal} \mathrm{mol}^{-1} \\
3 \mathrm{~B}_{10}+3 \mathrm{La}+\mathrm{e}^{-} \rightarrow 3 \mathrm{~B}_{4}+\mathrm{La}_{3} \mathrm{~B}_{18}^{-} \quad \Delta E_{\mathrm{A}}=-604.5 \mathrm{kcal} \mathrm{mol}^{-1}
\end{gathered}
$$

The energetics were calculated from single-point energy differences of the reactants and products, using the geometries directly taken from the optimized $\mathrm{La}_{3} \mathrm{~B}_{18}{ }^{-}$cage at the PBE/TZP level of theory. The interactions between the $\mathrm{B}_{18}$ framework and the three La atoms are extremely strong (steps 2 and 3), which underlies the stability of the $\mathrm{La}_{3} \mathrm{~B}_{18}{ }^{-}$cage. It should be noted that in platonic solids four surfaces are the minimum number to form a $3 \mathrm{D}$ object, i.e., the tetrahedron. However, Fig. 5a shows that three $\mathrm{La} @ \mathrm{~B}_{10}$ surfaces are fused together to form the $D_{3 h} \mathrm{La}_{3} \mathrm{~B}_{18}{ }^{-}$ cage. This is because the $\mathrm{La} \odot \mathrm{B}_{10}$ surface is curved. It turns out that the $\mathrm{Ln}_{3} \mathrm{~B}_{18}{ }^{-}$metallo-borospherenes belong to a class of geometry mathematically known as $n$-gonal hosohedron, which is basically a tessellation of lunes on a spherical surface, such that each lune shares the same two vertices. Thus, the $D_{3 h} \mathrm{La}_{3} \mathrm{~B}_{18}$ cage is a trigonal hosohedron, also known as spherical trihedron, where the two vertices consist of the triangular $\mathrm{B}_{6}$ units. To the best of our knowledge, such a geometry has not been observed in any cluster or molecular systems heretofore. 


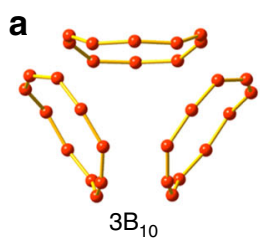

b

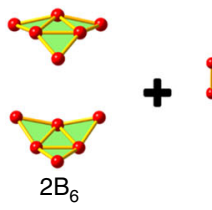

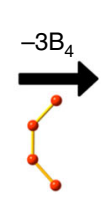

-

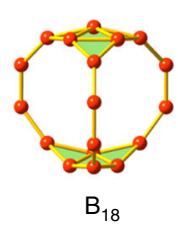

$\mathrm{B}_{18}$

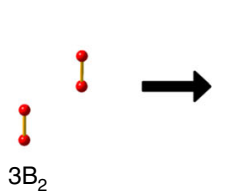

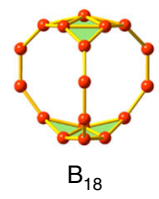
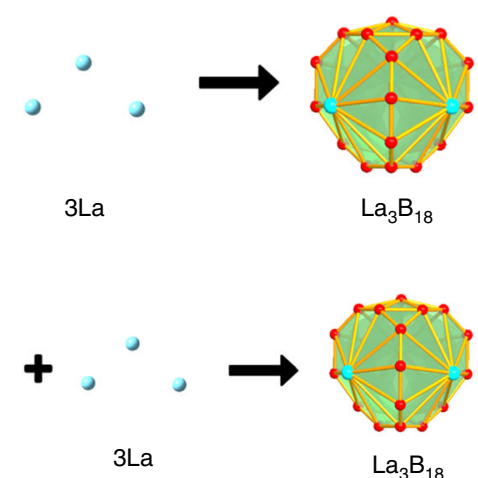

3La

3La

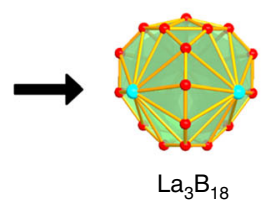

Fig. 5 Schematic pathways for the formation of the $D_{\mathbf{3 h}} \mathbf{L n}_{\mathbf{3}} \mathbf{B}_{\mathbf{1 8}}{ }^{-}$metallo-borospherenes. Two pathways for the construction of the $B_{18}$ framework and its bonding with the three $\mathrm{Ln}$ atoms are depicted. $\mathbf{a}$ The fused $\mathrm{B}_{10}$ ring pathway. $\mathbf{b}$ The $\mathrm{B}_{2}$-linked triangular $\mathrm{B}_{6}$ pathway.

Figure $5 \mathrm{~b}$ shows another pathway to construct the $\mathrm{La}_{3} \mathrm{~B}_{18}{ }^{-}$ cage, in which the $\mathrm{B}_{18}$ framework is formed by two triangular $\mathrm{B}_{6}$ motifs linked by three $B_{2}$ bridges, such that three $B_{10}$ rings are created. This hypothetical pathway can be represented by the following steps:

$$
\begin{gathered}
2 \mathrm{~B}_{6}+3 \mathrm{~B}_{2} \rightarrow \mathrm{B}_{18} \quad \Delta E_{5}=-707.9 \mathrm{kcal} \mathrm{mol}^{-1} \\
\mathrm{~B}_{18}+3 \mathrm{La} \rightarrow \mathrm{La}_{3} \mathrm{~B}_{18} \quad \Delta E_{6}=-655.8 \mathrm{kcal} \mathrm{mol}^{-1} \\
\mathrm{La}_{3} \mathrm{~B}_{18}+\mathrm{e}^{-} \rightarrow \mathrm{La}_{3} \mathrm{~B}_{18}^{-} \quad \Delta E_{7}=-68.8 \mathrm{kcal} \mathrm{mol}^{-1} \\
2 \mathrm{~B}_{6}+3 \mathrm{~B}_{2}+3 \mathrm{La}+e^{-} \rightarrow \mathrm{La}_{3} \mathrm{~B}_{18}^{-} \quad \Delta E_{B}=-1432.5 \mathrm{kcal} \mathrm{mol}^{-1}
\end{gathered}
$$

The interactions between the $\mathrm{B}_{18}$ framework and the three $\mathrm{La}$ atoms to form the $\mathrm{La}_{3} \mathrm{~B}_{18}{ }^{-}$cage are represented by steps $2 / 3$ or $6 / 7$ with an estimated binding energy of $724.6 \mathrm{kcal} \mathrm{mol}^{-1}$, i.e., $241.5 \mathrm{kcal} \mathrm{mol}^{-1}$ for the binding energy between each La atom and the $\mathrm{B}_{10}$ ring. This huge La- $\mathrm{B}_{10}$ binding energy underlies the extraordinary stability of the $\mathrm{La}_{3} \mathrm{~B}_{18^{-}}$metallo-borospherene. Compared with the pathway in Fig. 5a, the pathway in Fig. 5b is more favorable energetically since each step is exothermic. We should emphasize, though, that these exercises provide different views of the unprecedented hollow cage structures. The two pathways to construct the $\mathrm{La}_{3} \mathrm{~B}_{18}{ }^{-}$cage depicted in Fig. 5 certainly do not represent the mechanisms about how it is formed.

The nature of the bonding between $B_{18}$ and the La atoms. Since the global minimum of $B_{18}$ is a planar structure ${ }^{36}$, the stabilization of the $3 \mathrm{D} \mathrm{B}_{18}$ framework is entirely due to its strong bonding with the three La atoms, as discussed above. We have analyzed the nature of the $\mathrm{La}-\mathrm{B}_{10}$ bonding in the $\mathrm{La}_{3} \mathrm{~B}_{18}{ }^{-}$metalloborospherene using several different methods. The MO energylevel diagram and the relevant MOs of $\mathrm{La}_{3} \mathrm{~B}_{18}{ }^{-}$derived from the $\mathrm{La}_{3}{ }^{-}$and $\mathrm{B}_{18}$ moieties are shown in Fig. 4 . The $5 e^{\prime \prime}, 9 e^{\prime}$, and $1 a_{1}{ }^{\prime \prime}$ MOs of $\mathrm{La}_{3} \mathrm{~B}_{18}{ }^{-}$(red-colored) represent the bonding orbitals between the three $\mathrm{La}$ atoms and the $\mathrm{B}_{18}$ moiety, mainly corresponding to the interactions between the irreducible representations, $4 e^{\prime \prime}, 6 e^{\prime}$, and $1 a_{1}{ }^{\prime \prime}$ on the $\mathrm{B}_{18}$ moiety and $6 e^{\prime \prime}, 12 e^{\prime}$, and $2 a_{1}{ }^{\prime \prime}$ on the $\mathrm{La}_{3}{ }^{-}$moiety (the red highlighted MOs). Supplementary Table S5 gives the compositions of the $5 e^{\prime \prime}, 9 e^{\prime}$, and $1 a_{1}^{\prime \prime}$ MOs, which are dominated by contributions from the $\mathrm{B}_{18}$ moiety. Hence, there is a strong charge transfer from $\mathrm{La}$ to $\mathrm{B}_{18}$, resulting in a closed-shell $\mathrm{La}_{3} \mathrm{~B}_{18}{ }^{-}$with a large HOMO-LUMO gap of $1.51 \mathrm{eV}$ computed at the PBE/TZP level. The La atoms are in their favorite + III oxidation state in $\mathrm{La}_{3} \mathrm{~B}_{18}{ }^{-}$, which can be viewed approximately as $\left(\mathrm{La}^{3+}\right)_{3}\left[\mathrm{~B}_{18}{ }^{10-}\right]$. As shown in Fig. 4, the $6 e^{\prime \prime}$, $12 e^{\prime}$, and $2 a_{1}^{\prime \prime}$ irreducible representations on the $\mathrm{La}_{3}{ }^{-}$moiety are of La $5 d$ characters, while the $4 e^{\prime \prime}, 6 e^{\prime}$, and $1 a_{1}^{\prime \prime}$ irreducible representations on the $\mathrm{B}_{18}$ framework are of $\mathrm{B} 2 p$ characters. Hence, the $5 e^{\prime \prime}, 9 e^{\prime}$, and $1 a_{1}{ }^{\prime \prime}$ MOs also represent significant La $5 d$ and $\mathrm{B}_{18} 2 p$ covalent bonding. It is the strong covalent and ionic bonding between the $\mathrm{La}$ atoms and the $\mathrm{B}_{10}$ rings that gives rise to the extraordinary stability of the $\mathrm{La}_{3} \mathrm{~B}_{18}{ }^{-}$cage structure. These bonding characteristics are found in all lanthanide boride compounds due to the low electronegativity of the lanthanide elements and their diffuse $5 d$ orbitals $^{37}$.

The $\mathrm{La}-\mathrm{B}_{10}$ interactions can be further characterized using the EDA-NOCV method with $\mathrm{B}_{18}\left(\ldots 6 a_{1}{ }^{2} 1 a_{1}{ }^{\prime \prime} 6 e^{\prime 0} 4 e^{\prime \prime 0}\right)$ and $\mathrm{La}_{3}{ }^{-}$ $\left(\ldots 6 e^{\prime \prime 4} 2 a_{1}{ }^{\prime \prime 2} 12 e^{\prime 4}\right)$ fragments, a powerful energy decomposition tool to give insight into chemical bonding ${ }^{38}$. We analyzed the $\mathrm{B}_{18} \ldots \mathrm{La}_{3}{ }^{-}$interaction by the decomposition of the orbital terms into pairwise contributions, as shown in Supplementary Fig. 6 . There are three major terms $\Delta E_{1}, \Delta E_{2}$, and $\Delta E_{3}$ associated with the deformation densities $\Delta \rho_{1}, \Delta \rho_{3}$, and $\Delta \rho_{3}$, respectively. The remaining terms contribute $<10 \%$ to the total orbital interactions. The color code of the deformation densities indicates the direction of the charge flow from red $\rightarrow$ blue. It is interesting to see that the $1 a_{1}{ }^{\prime \prime}$ orbital of $\mathrm{La}_{3} \mathrm{~B}_{18}^{-}$, which is analogous to the $(d-p) \delta$ bonding $\mathrm{MO}$ in the $\operatorname{Ln}_{2} \mathrm{~B}_{8}{ }^{-}$inverse sandwich complexes $^{31,32}$, contributes significantly (25.7\% from the EDANOCV analyses, Supplementary Fig. 6) to the stability of the orbital interaction. The other two stronger $\Delta \rho_{2}(34.8 \%)$ and $\Delta \rho_{3}$ (28.9\%) deformation densities correspond to the degenerate $9 e^{\prime}$ and $5 e^{\prime \prime}$ MOs, respectively. The direction of the charge flow is from the $\mathrm{La}_{3}{ }^{-}$to the $\mathrm{B}_{18}$ moiety, consistent with the fragment $\mathrm{MO}$ analyses discussed above (Fig. 4).

We further analyzed the chemical bonding in the $\mathrm{La}_{3} \mathrm{~B}_{18}{ }^{-}$ metallo-borospherene using the adaptive natural density partitioning (AdNDP) approach ${ }^{39}$, as shown in Fig. 6. The first row displays nine localized two-center two-electron $(2 \mathrm{c}-2 \mathrm{e}) \sigma$ bonds formed within the three $B_{2}$ units and between the $B_{2}$ units and the three apexes of the two $B_{6}$ triangles. The second row reveals the delocalized bonds in the $\mathrm{B}_{6}$ triangles, with four three-center twoelectron (3c-2e) $\sigma$ bonds within each $\mathrm{B}_{6}$ unit. The multi-center $12 \mathrm{c}-2 \mathrm{e}$ and $18 \mathrm{c}-2 \mathrm{e}$ delocalized bonds can be viewed as $\pi$ bonds within the $\mathrm{B}_{6}$ units. The third row represents totally delocalized $\sigma$ and $\pi$ bonds within the $B_{18}$ framework. The last row shows five totally delocalized $21 \mathrm{c}-2 \mathrm{e}$ bonds between the La atoms and the $\mathrm{B}_{18}$ framework, corresponding to the $5 e^{\prime \prime}, 9 e^{\prime}$, and $1 a_{1}{ }^{\prime \prime}$ MOs in Fig. 4. We also found that the $\mathrm{La}_{3} \mathrm{~B}_{18}{ }^{-}$metallo-borospherene possesses both $3 \mathrm{D}$ aromaticity with calculated nucleusindependent chemical shifts (NICS) ${ }^{40}$ of $-47.87 \mathrm{ppm}$ at the cage center, and planar aromaticity on each $\mathrm{B}_{6}$ triangles with NICS $(0)$ of $-31.44 \mathrm{ppm}$ and NICS(1) of -2.16 above the plane center, as shown in Supplementary Table 6, where the aromaticity in the 


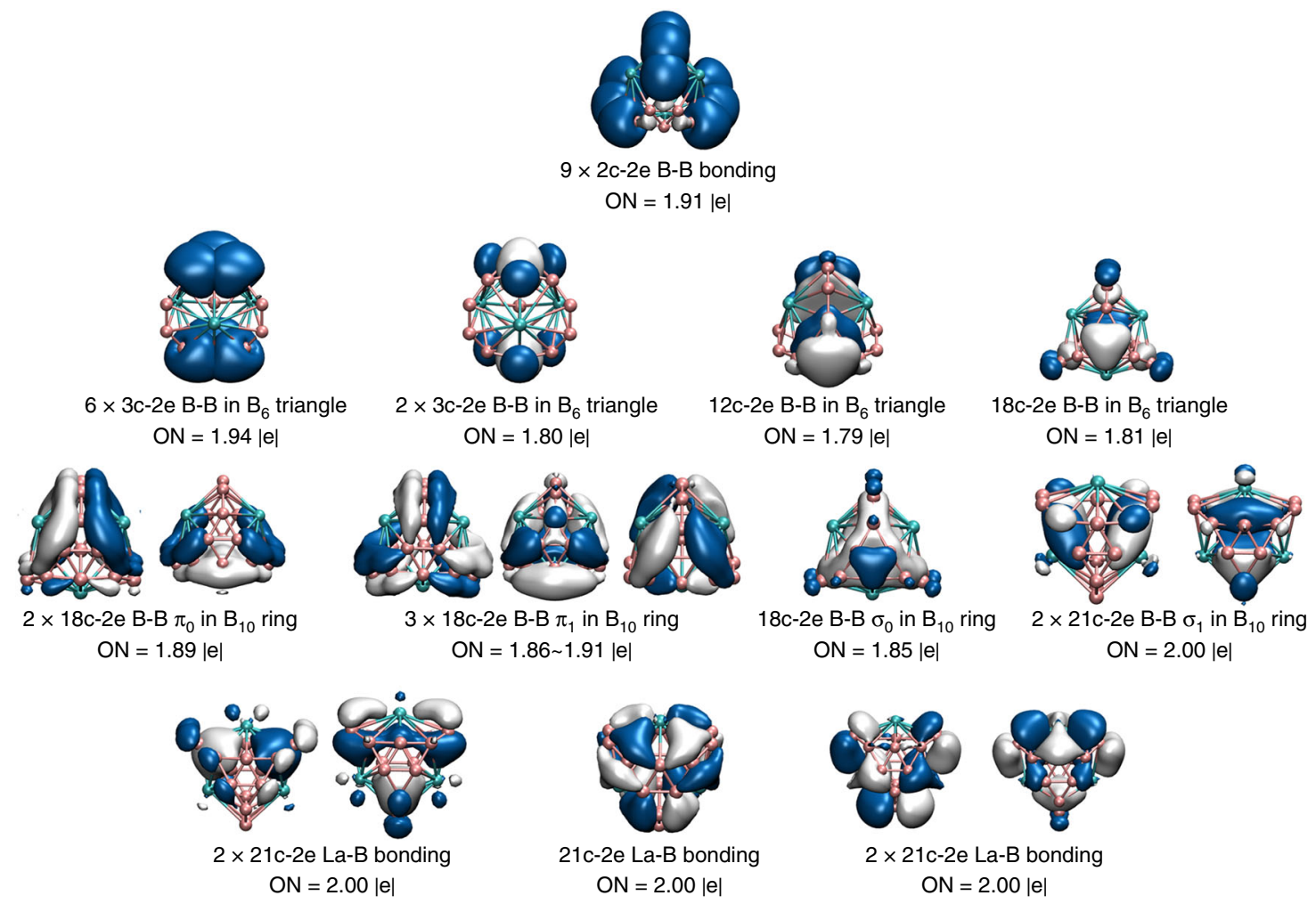

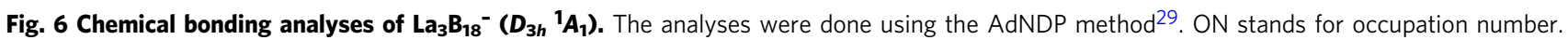

metallo-borospherene is compared with that of the recently synthesized cubic $\left[\mathrm{Zn}^{\mathrm{I}}\right]_{8}$ compound ${ }^{41}$.

We also performed bond-order index analyses for the B-B and La-B interactions, as presented in Supplementary Table 7. The $\mathrm{B}_{2}$ bridges have shorter bond lengths and higher bond orders than those of the $\mathrm{B}_{6}$ triangles. In terms of the La-B interactions, the distances and bond order indices are similar to those in the lanthanide-boron complexes reported previously $y^{30-33}$.

A new class of spherical trihedron metallo-borospherenes. The discoveries of the $\mathrm{La}_{3} \mathrm{~B}_{18}{ }^{-}$and $\mathrm{Tb}_{3} \mathrm{~B}_{18^{-}}$metallo-borospherenes suggest that other lanthanide elements could also form similar structures because of the similarity in the chemical properties of the whole series of lanthanides. We have calculated the $D_{3 h}$ cage structures for all the lanthanide elements, $\operatorname{Ln}_{3} \mathrm{~B}_{18}{ }^{-}(\mathrm{Ln}=\mathrm{Ce}-\mathrm{Lu})$. The coordinates obtained at the PBE0 level are given as Supplementary Data 1 , whereas those of $\mathrm{La}_{3} \mathrm{~B}_{18}{ }^{-}$and $\mathrm{La}_{3} \mathrm{~B}_{18}$ are provided in Supplementary Table 8. All these structures are indeed minima on their potential energy surfaces. Hence, we conclude that there indeed exist a whole class of $\mathrm{Ln}_{3} \mathrm{~B}_{18}{ }^{-}$metalloborospherenes. While borospherenes have not been observed beyond the $\mathrm{B}_{40}$ cluster ${ }^{42}$, the unique bonding characteristics between lanthanide and boron suggest that other lanthanide metallo-borospherenes with different sizes and $\operatorname{Ln}_{x} B_{y}{ }^{-}$stoichiometries may exist. Recent studies of transition-metal borides showed that the metal-boron interactions have major influences on their magnetic properties ${ }^{43,44}$. Hence, the understanding of Ln-B interactions in the metallo-borospherene systems may provide insights for the design of lanthanide borides with tunable magnetic or catalytic properties.

In conclusion, we report the observation of the first trilanthanide-doped boron cage clusters (metallo-borospherenes), in which the metal atoms are integral parts of the cage surface. Photoelectron spectra of two representative systems, $\operatorname{Ln}_{3} \mathrm{~B}_{18}{ }^{-}$ $(\mathrm{Ln}=\mathrm{La}, \mathrm{Tb})$, show similar and relatively simple spectral patterns, suggesting that they have similar highly symmetric structures. Theoretical calculations reveal that the $\mathrm{Ln}_{3} \mathrm{~B}_{18}{ }^{-}$anions have cagelike structures with $D_{3 h}$ symmetry: two planar $\mathrm{B}_{6}$ triangular units linked by three $\mathrm{B}_{2}$ bridges to form the $\mathrm{B}_{18}$ framework consisting of three shared $\mathrm{B}_{10}$ rings coordinated to the three $\mathrm{Ln}$ atoms. Strong ionic and covalent chemical bonding is found between the Ln atoms and the $\mathrm{B}_{18}$ framework. The extraordinary stabilities of the metallo-borospherenes are understood by various theoretical analyses. $\mathrm{La}_{3} \mathrm{~B}_{18}{ }^{-}$is found to have a closed-shell electron configuration with a large HOMO-LUMO gap and possesses $3 \mathrm{D}$ aromaticity. The $\mathrm{Ln}_{3} \mathrm{~B}_{18}{ }^{-}$cage complexes are expected to exist for all lanthanide elements, suggesting the possibility that there may exist a large class of lanthanide metallo-borospherenes with different Ln/B stoichiometries and tunable properties.

\section{Methods}

Experimental details. The experiments were carried out using a magnetic-bottle PES apparatus equipped with a laser vaporization supersonic cluster source, details of which have been published elsewhere ${ }^{11}$. The $\mathrm{La}_{3} \mathrm{~B}_{18}{ }^{-}$and $\mathrm{Tb}_{3} \mathrm{~B}_{18}{ }^{-}$clusters were produced by laser vaporization of a $\mathrm{La} /{ }^{11} \mathrm{~B}$ or $\mathrm{Tb} /{ }^{11} \mathrm{~B}$ mixed target, respectively. The laser-induced plasma was cooled by a He carrier gas seeded with $5 \% \mathrm{Ar}$, initiating nucleation between the boron and lanthanide atoms. The nascent clusters were entrained in the carrier gas and underwent a supersonic expansion. Negatively-charged clusters were extracted from the collimated cluster beam and analyzed by a time-of-flight mass spectrometer. Both pure $\left(\mathrm{B}_{n}^{-}\right)$and mixed $\left(\operatorname{Ln}_{x} \mathrm{~B}_{y}{ }^{-}\right)$clusters were produced from the cluster source. The $\mathrm{La}_{3} \mathrm{~B}_{18}{ }^{-}$and $\mathrm{Tb}_{3} \mathrm{~B}_{18}$ clusters of current interest were mass-selected and photodetached by the $193 \mathrm{~nm}$ $(6.424 \mathrm{eV})$ radiation from an ArF excimer laser or the fourth harmonics from a $\mathrm{Nd}$ : YAG laser $(266 \mathrm{~nm}, 4.661 \mathrm{eV})$. Photoelectrons were collected and analyzed in a 3.5 $\mathrm{m}$-long electron flight tube at nearly $100 \%$ efficiency. The photoelectron spectra were calibrated by the known transitions of $\mathrm{Au}^{-}$and $\mathrm{Bi}^{-}$. The resolution of the PES apparatus $(\triangle \mathrm{KE} / \mathrm{KE})$ was around $2.5 \%$, that is, about $25 \mathrm{meV}$ for photoelectrons with $1 \mathrm{eV}$ kinetic energy (KE).

Computational methods. Unbiased global-minimum structural searches for the $\mathrm{La}_{3} \mathrm{~B}_{18}{ }^{-}$cluster were performed using the TGMin 2.0 code $^{45}$. The global minimum structure of $\mathrm{Tb}_{3} \mathrm{~B}_{18}{ }^{-}$was not searched separately. More than 2000 structures were evaluated for $\mathrm{La}_{3} \mathrm{~B}_{18}{ }^{-}$using the constrained Basin-Hopping algorithm at the PBE/ $\mathrm{DZP}^{46,47}$ level from the ADF 2017 software ${ }^{48}$. A $D_{3 h}$ cage structure was found to be 
the global minimum, which was significantly lower in energy in comparison to the next lowest-lying isomer (Supplementary Fig. 2). To confirm the stability of the global minimum, we conducted another 500 structural searches, using the $D_{3 h}$ cage as the seed structure. No structures with lower energies were found. All the local minima were verified via harmonic vibrational frequency calculations. The frozencore approximation was employed for the inner shells of $\left[1 s^{2}\right]$ for B and $\left[1 s^{2}-4 d^{10}\right]$ for the La atoms. The zero-order regular approximation ${ }^{49}$ was applied, to account for the scalar relativistic effects. Low-lying isomers were subsequently optimized using the PBE and PBE0 density functionals ${ }^{50}$ along with the TZP basis sets. Born-Oppenheimer molecular dynamic simulations were further carried out on $\mathrm{La}_{3} \mathrm{~B}_{18}{ }^{-}$for $13 \mathrm{ps}$ using the $\mathrm{CP} 2 \mathrm{~K}$ code ${ }^{51}$ at different temperatures, from 300 to $1000 \mathrm{~K}$ (Supplementary Fig. 4). To minimize the 4 -electron induced complexity (i.e. spin multiplicity) and considering the negligible geometry change due to the occupations of the localized $4 f$ orbitals (radial-density maximum probability radii $<0.5 \AA)$, we used the $4 f$-in-core pseudopotentials ${ }^{34}$ for the lanthanide elements to optimize the geometric parameters in the other $\mathrm{Ln}_{3} \mathrm{~B}_{18}{ }^{-}(\mathrm{Ln}=$ $\mathrm{Ce}-\mathrm{Lu})$ species.

The simulation of the PE spectra was done using the $\triangle$ SCF-TDDFT method ${ }^{52}$ with the SAOP exchange-correlation functional ${ }^{53}$ to account for the long-range interactions. The ground state adiabatic and vertical detachment energies were calculated at the DFT levels, as well as the more accurate DLPNO-CCSD(T) level ${ }^{54}$ with the Def2-TZVP basis sets ${ }^{55}$ and the Def2-TZVPP pseudopotential for La ${ }^{34}$ utilizing the AutoAux generation procedure ${ }^{55}$. We also used the 4 -in-core pseudopotential ${ }^{34}$ for the simulation of the PE spectrum of $\mathrm{Tb}_{3} \mathrm{~B}_{18}{ }^{-}$without consideration of the $4 f$ electron detachment channels. Previous studies showed that such detachment channels carried very low detachment cross sections at the low detachment photon energies used and the main PES features of Ln-B binary clusters were dominated by MOs with Ln $s d$ or B $s p$ characters ${ }^{31-33}$. Chemical bonding and electronic structure analyses were carried out by canonical molecular orbital (MO) theory and the semi-localized AdNDP method ${ }^{39}$. We also performed calculations using the energy decomposition analysis-natural orbitals for chemical valence (EDA-NOCV) approach ${ }^{38}$ to quantitatively elucidate the bonding mechanisms between the $\mathrm{B}_{18}$ and $\mathrm{La}_{3}{ }^{-}$moieties. The bond order indexes of different interatomic interactions were calculated using the $\mathrm{Mayer}^{56}$, Gopinathan-Jug $(\mathrm{G}-\mathrm{J})^{57}$, and Nalewajski-Mrozek schemes ${ }^{58}$.

\section{Data availability}

The data that support the findings of this study are available within the article and the associated Supplementary information. Any other data are available from the corresponding authors upon request.

\section{Code availability}

The TGMin code used for the global minimum search is available from the corresponding author (J.L.) upon request and signing a license.

Received: 14 January 2020; Accepted: 4 May 2020;

Published online: 02 June 2020

\section{References}

1. Lipscomb, W. N. The boranes and their relatives. Science 196, 1047-1055 (1977).

2. Oganov, A. R. et al. Ionic high-pressure form of elemental boron. Nature 457, 863-866 (2009).

3. Albert, B. \& Hillebrecht, H. Boron: elementary challenge for experimenters and theoreticians. Angew. Chem. Int. Ed. 48, 8640-8668 (2009).

4. Gindulyte, A., Lipscomb, W. N. \& Massa, L. Proposed boron nanotubes. Inorg. Chem. 37, 6544-6545 (1998).

5. Boustani, I., Quandt, A., Hernández, E. \& Rubio, A. New boron based nanostructured materials. J. Chem. Phys. 110, 3176-3185 (1999).

6. Tang, H. \& Ismail-Beigi, S. Novel precursors for boron nanotubes: the competition of two-center and three-center bonding in boron sheets. Phys. Rev. Lett. 99, 115501 (2007).

7. Yang, X., Ding, Y. \& Ni, J. Ab initio prediction of stable boron sheets and boron nanotubes: structure, stability, and electronic properties. Phys. Rev. B 77, 041402 (2008).

8. Zhai, H. J., Kiran, B., Li, J. \& Wang, L. S. Hydrocarbon analogs of boron clusters: Planarity, aromaticity, and antiaromaticity. Nat. Mater. 2, 827-833 (2003).

9. Alexandrova, A. N., Boldyrev, A. I., Zhai, H. J. \& Wang, L. S. All-boron aromatic clusters as potential new inorganic ligands and building blocks in chemistry. Coord. Chem. Rev. 250, 2811-2866 (2006).

10. Sergeeva, A. P. et al. Understanding boron through size-selected clusters: structure, chemical bonding, and fluxionality. Acc. Chem. Res. 47, 1349-1358 (2014).
11. Wang, L. S. Photoelectron spectroscopy of size-selected boron clusters: from planar structures to borophenes and borospherenes. Int. Rev. Phys. Chem. 35, 69-142 (2016).

12. Piazza, $\mathrm{Z}$. A. et al. Planar hexagonal $\mathrm{B}_{36}$ as a potential basis for extended single-atom layer boron sheets. Nat. Commun. 5, 3113 (2014).

13. Mannix, A. J. et al. Synthesis of borophenes: anisotropic, two-dimensional boron polymorphs. Science 350, 1513-1516 (2015).

14. Feng, B. et al. Experimental realization of two-dimensional boron sheets. Nat. Chem. 8, 563 (2016).

15. Mannix, A. J., Zhang, Z., Guisinger, N. P., Yakobson, B. I. \& Hersam, M. C. Borophene as a prototype for synthetic 2D materials development. Nat. Nanotechnol. 13, 444 (2018).

16. Zhai, H. J. et al. Observation of an all-boron fullerene. Nat. Chem. 6, 727 (2014).

17. Chen, Q. et al. Experimental and theoretical evidence of an axially chiral borospherene. ACS Nano 9, 754-760 (2014).

18. Lu, X., Feng, L., Akasaka, T. \& Nagase, S. Current status and future developments of endohedral metallofullerenes. Chem. Soc. Rev. 41, 7723-7760 (2012).

19. Zhang, $\mathrm{X}$. et al. A diuranium carbide cluster stabilized inside a $\mathrm{C}_{80}$ fullerene cage. Nat. Commun. 9, 2753 (2018).

20. Clemmer, D. E., Hunter, J. M., Shelimov, K. B. \& Jarrold, M. F. Physical and chemical evidence for metallofullerenes with metal atoms as part of the cage. Nature 372, 248-250 (1994)

21. Branz, W. et al. Cage substitution in metal-fullerene clusters. J. Chem. Phys. 109, 3425-3430 (1998).

22. Vostrowsky, O. \& Hirsch, A. Heterofullerenes. Chem. Rev. 106, 5191-5207 (2006).

23. Guo, B., Kerns, K. \& Castleman, A. $\mathrm{Ti}_{8} \mathrm{C}_{12}{ }^{+}$-metallo-carbohedrenes: a new class of molecular clusters? Science 255, 1411-1413 (1992).

24. Dance, $\mathrm{I}_{\text {. }} \mathrm{Ti}_{8} \mathrm{C}_{12}$ : barrierless transformation of the $T_{h}$ Isomer to the $T_{d}$ isomer. J. Am. Chem. Soc. 118, 6309-6310 (1996).

25. Romanescu, C., Galeev, T. R., Li, W. L., Boldyrev, A. I. \& Wang, L. S. Aromatic metal-centered monocyclic boron rings: $\mathrm{Co} \odot \mathrm{B}_{8}{ }^{-}$and $\mathrm{Ru}^{\circ} \mathrm{B}_{9}{ }^{-}$. Angew. Chem. Int. Ed. 50, 9334-9337 (2011).

26. Romanescu, C., Galeev, T. R., Li, W. L., Boldyrev, A. I. \& Wang, L. S. Transition-metal-centered monocyclic boron wheel clusters $\left(\mathrm{M} \odot \mathrm{B}_{\mathrm{n}}\right)$ : a new class of aromatic borometallic compounds. Acc. Chem. Res. 46, 350-358 (2012).

27. Popov, I. A., Jian, T., Lopez, G. V., Boldyrev, A. I. \& Wang, L. S. Cobaltcentred boron molecular drums with the highest coordination number in the $\mathrm{CoB}_{16}-$ cluster. Nat. Commun. 6, 8654 (2015)

28. Li, W. L. et al. The planar $\mathrm{CoB}_{18}{ }^{-}$cluster as a motif for metallo-borophenes. Angew. Chem. Int. Ed. 55, 7358-7363 (2016).

29. Li, W. L. et al. From planar boron clusters to borophenes and metalloborophenes. Nat. Rev. Chem. 1, 0071 (2017).

30. Jian, T. et al. Probing the structures and bonding of size-selected boron and doped-boron clusters. Chem. Soc. Rev. 48, 3550-3591 (2019).

31. Li, W. L. et al. Observation of highly stable and symmetric lanthanide octaboron inverse sandwich clusters. Proc. Natl Acad. Sci. USA 115, E6972-E6977 (2018).

32. Chen, T. T., Li, W. L., Li, J. \& Wang, L. S. La ( $7 x-B x) L a]-(x=7-9)$ : a new class of inverse sandwich complexes.Chem. Sci. 10, 2534-2542 (2019).

33. Chen, T. T., Li, W. L., Chen, W. J., Li, J. \& Wang, L. S. $\mathrm{La}_{3} \mathrm{~B}_{14^{-}}{ }^{-}$an inverse triple-decker lanthanide boron cluster. Chem. Commun. 55, 7864-7867 (2019).

34. Dolg, M., Stoll, H. \& Preuss, H. A combination of quasi-relativistic pseudopotential and ligand field calculations for lanthanoid compounds. Theor. Chim. Acta 85, 441-450 (1993).

35. Malik, Z. P., Sologub, O., Grytsiv, A., Giester, G. \& Rogl, P. F. Crystal structure of novel Ni-Zn borides: first observation of a boron-metal nested cage unit: $\mathrm{B}_{20} \mathrm{Ni}_{6}$. Inorg. Chem. 50, 7669-7675 (2011).

36. Sergeeva, A. P., Averkiev, B. B., Zhai, H. J., Boldyrev, A. I. \& Wang, L. S. Allboron analogues of aromatic hydrocarbons: $\mathrm{B}_{17}{ }^{-}$and $\mathrm{B}_{18}{ }^{-}$. J. Chem. Phys. 134, 224304 (2011).

37. Scheifers, J. P., Zhang, Y. \& Fokwa, B. P. Boron: Enabling exciting metal-rich structures and magnetic properties. Acc. Chem. Res. 50, 2317-2325 (2017)

38. Mitoraj, M. P., Michalak, A. \& Ziegler, T. A combined charge and energy decomposition scheme for bond analysis. J. Chem. Theory Comput. 5, 962-975 (2009).

39. Zubarev, D. Y. \& Boldyrev, A. I. Developing paradigms of chemical bonding: adaptive natural density partitioning. Phys. Chem. Chem. Phys. 10, 5207-5217 (2008).

40. Schleyer, Pv. R., Maerker, C., Dransfeld, A., Jiao, H. \& van Eikema Hommes, N. J. Nucleus-independent chemical shifts: a simple and efficient aromaticity probe. J. Am. Chem. Soc. 118, 6317-6318 (1996).

41. Cui, P. et al. A multicentre-bonded $\left[\mathrm{Zn}^{\mathrm{I}}\right]_{8}$ cluster with cubic aromaticity. Nat. Commun. 6, 6331 (2015). 
42. Bai, $\mathrm{H}$. et al. Planar $\mathrm{B}_{41}{ }^{-}$and $\mathrm{B}_{42}{ }^{-}$clusters with double-hexagonal vacancies. Nanoscale 11, 23286-23295 (2019).

43. Mbarki, M., St. Touzani, R. \& Fokwa, B. P. Unexpected synergy between magnetic iron chains and stacked $\mathrm{B}_{6}$ rings in $\mathrm{Nb}_{6} \mathrm{Fe}_{1-\mathrm{x}} \mathrm{Ir}_{6+\mathrm{x}} \mathrm{B}_{8}$. Angew. Chem. Int. Ed. 53, 13174-13177 (2014).

44. Sharma, N., Zhang, Y., Fokwa, B., Mbarki, M. \& Huq, A. Structural-distortiondriven magnetic transformation from ferro-to ferrimagnetic iron chains in $\mathrm{B}_{6}$ based $\mathrm{Nb}_{6} \mathrm{FeIr}_{6} \mathrm{~B}_{8}$. Angew. Chem. Int. Ed. 57, 10323-10327 (2018).

45. Zhao, Y., Chen, X. \& Li, J. TGMin: a global-minimum structure search program based on a constrained basin-hopping algorithm. Nano Res. 10, 3407-3420 (2017).

46. Perdew, J. P., Burke, K. \& Ernzerhof, M. Generalized gradient approximation made simple. Phys. Rev. Lett. 77, 3865-3868 (1996).

47. Van Lenthe, E. \& Baerends, E. J. Optimized Slater-type basis sets for the elements 1-118. J. Comput. Chem. 24, 1142-1156 (2003).

48. ADF, SCM, Theoretical Chemistry, Vrijie Universiteit, (Amsterdam, The Netherlands, 2017). http://www.scm.com.

49. Lenthe, E. V., Baerends, E.-J. \& Snijders, J. G. Relativistic regular twocomponent Hamiltonians. J. Chem. Phys. 99, 4597-4610 (1993).

50. Adamo, C. \& Barone, V. Toward reliable density functional methods without adjustable parameters: the PBE0 model. J. Chem. Phys. 110, 6158-6170 (1999).

51. VandeVondele, J. et al. Quickstep: fast and accurate density functional calculations using a mixed Gaussian and plane waves approach. Comput. Phys. Commun. 167, 103-128 (2005).

52. Li, J., Li, X., Zhai, H. J. \& Wang, L. S. Au ${ }_{20}$ : a tetrahedral cluster. Science 299, 864-867 (2003).

53. Schipper, P. R., Gritsenko, O. V., van Gisbergen, S. J. \& Baerends, E. J. Molecular calculations of excitation energies and (hyper) polarizabilities with a statistical average of orbital model exchange-correlation potentials. J. Chem. Phys. 112, 1344-1352 (2000).

54. Neese, F., Hansen, A. \& Liakos, D. G. Efficient and accurate approximations to the local coupled cluster singles doubles method using a truncated pair natural orbital basis. J. Chem. Phys. 131, 064103 (2009).

55. Weigend, F. \& Ahlrichs, R. Balanced basis sets of split valence, triple zeta valence and quadruple zeta valence quality for $\mathrm{H}$ to $\mathrm{Rn}$ : design and assessment of accuracy. Phys. Chem. Chem. Phys. 7, 3297-3305 (2005).

56. Mayer, I. Bond orders and valences from ab initio wave functions. Int. J. Quantum Chem. 29, 477-483 (1986).

57. Gopinathan, M. \& Jug, K. Valency. I. A quantum chemical definition and properties. Theor. Chim. Acta 63, 497-509 (1983).

58. Michalak, A., DeKock, R. L. \& Ziegler, T. Bond multiplicity in transition-metal complexes: applications of two-electron valence indices. J. Phys. Chem. A 112, 7256-7263 (2008).

\section{Acknowledgements}

The experiment done at Brown University was supported by the National Science Foundation (CHE-1763380). The theoretical work done at Tsinghua University was supported by the National Natural Science Foundation of China (21590792, 91426302, and 21433005). The calculations were done using supercomputers at the Southern University of Science and Technology (SUSTech), Tsinghua National Laboratory for Information Science and Technology, and the Computational Chemistry Laboratory of the Department of Chemistry under the Tsinghua Xuetang Talents Program.

\section{Author contributions}

T-T.C. and W-J.C. conducted the experiment; W-L.L. did the calculations with assistance from X-H.Y. and X-R.D.; J.L. and L-S.W. guided the work. T-T.C., W-L.L., J.L., and L-S.W. co-wrote the manuscript. All the authors read and commented on the manuscript.

\section{Competing interests}

The authors declare no competing interests.

\section{Additional information}

Supplementary information is available for this paper at https://doi.org/10.1038/s41467020-16532-x.

Correspondence and requests for materials should be addressed to W.-L.L., J.L. or L.-S.W.

Peer review information Nature Communications thanks Boniface Fokwa, Panaghiotis Karamanis and the other, anonymous, reviewer(s) for their contribution to the peer review of this work.

Reprints and permission information is available at http://www.nature.com/reprints

Publisher's note Springer Nature remains neutral with regard to jurisdictional claims in published maps and institutional affiliations.

Open Access This article is licensed under a Creative Commons Attribution 4.0 International License, which permits use, sharing, adaptation, distribution and reproduction in any medium or format, as long as you give appropriate credit to the original author(s) and the source, provide a link to the Creative Commons license, and indicate if changes were made. The images or other third party material in this article are included in the article's Creative Commons license, unless indicated otherwise in a credit line to the material. If material is not included in the article's Creative Commons license and your intended use is not permitted by statutory regulation or exceeds the permitted use, you will need to obtain permission directly from the copyright holder. To view a copy of this license, visit http://creativecommons.org/ licenses/by/4.0/.

(C) The Author(s) 2020 